\title{
Polyphasic approach to the identification and characterization of aflatoxigenic strains of Aspergillus section Flavi isolated from peanuts and peanut-based products marketed in Malaysia
}

\begin{abstract}
Peanuts are widely consumed as the main ingredient in many local dishes in Malaysia. However, the tropical climate in Malaysia (high temperature and humidity) favours the growth of fungi from Aspergillus section Flavi, especially during storage. Most of the species from this section, such as A. flavus, A. parasiticus and A. nomius, are natural producers of aflatoxins. Precise identification of local isolates and information regarding their ability to produce aflatoxins are very important to evaluate the safety of food marketed in Malaysia. Therefore, this study aimed to identify and characterize the aflatoxigenic and nonaflatoxigenic strains of Aspergillus section Flavi in peanuts and peanut-based products. A polyphasic approach, consisting of morphological and chemical characterizations was applied to 128 isolates originating from raw peanuts and peanut-based products. On the basis of morphological characters, 127 positively identified as Aspergillus flavus, and the other as A. nomius. Chemical characterization revealed six chemotype profiles which indicates diversity of toxigenic potential. About 58.6\%, 68.5\%, and 100\% of the isolates are positive for aflatoxins, cyclopiazonic acid and aspergillic acid productions respectively. The majority of the isolates originating from raw peanut samples $(64.8 \%)$ were aflatoxigenic, while those from peanut-based products were less toxigenic (39.1\%). The precise identification of these species may help in developing control strategies for aflatoxigenic fungi and aflatoxin contamination in peanuts, especially during storage. These findings also highlight the possibility of the co-occurrence of other toxins, which could increase the potential toxic effects of peanuts.
\end{abstract}

Keyword: Peanuts; Aspergillus flavus; Aflatoxins; Cyclopiazonic acid 